\title{
Predação de ninhos de Trachemys dorbigni (Duméril \& Bibron) (Testudines, Emydidae) no extremo sul do Brasil
}

\author{
Fernanda A. Gonçalves ${ }^{1}$; Sonia Z. Cechin ${ }^{1} \&$ Alex Bager ${ }^{2}$
}

\begin{abstract}
${ }^{1}$ Laboratório de Herpetologia, Departamento de Biologia, Universidade Federal de Santa Maria. Faixa de Camobi, km 9, Campus Universitário, Prédio 17, Sala 1140, 97105-900 Santa Maria, Rio Grande do Sul, Brasil.

E-mail: f_anziliero@hotmail.com

${ }^{2}$ Universidade Estadual do Rio Grande do Sul. Avenida Assis Brasil 842, Centro, 95400-000 São Francisco de Paula, Rio Grande do Sul, Brasil.
\end{abstract}

\begin{abstract}
Nest predation of Trachemys dorbigni (Duméril \& Bibron) (Testudines, Emydidae) in Southern Brazil. Nests of the D'Orbigny's slider, Trachemys dorbigni, were monitored during the nesting season of 2005/2006 to evaluate predation rates; time variation on these rates; to identify predator species and their importance on nest destruction and the influence of nest dispersion on predation rates in Southern Brazil. Of the 58 monitored nests, $98 \%(n=57)$ were destroyed by predators. Predation events occurred primarily during the first 48 hours after oviposition and there was no time variation on predation rates. Using camera traps and direct observations we could identify six species of nest predators. There was not prevalence of any predator. Nest density did not influence predation rates, but there was a negative correlation between time after egg laying and predation. KEY WORDS. Camera traps; chelonian; D’Orbigny's slider; predator; Taim Ecological Station.
\end{abstract}

RESUMO. Ninhos da tartaruga tigre-d'água, Trachemys dorbigni, foram monitorados durante a estação reprodutiva de 2005/2006 para avaliar as taxas de predação e a variação temporal destas; identificar as espécies predadoras, sua importância na destruição dos ninhos e determinar a influência da dispersão dos ninhos sobre a predação, no extremo sul do Brasil. Dos 58 ninhos monitorados, 98\% $(n=57)$ foram destruídos por predadores. Eventos de predação ocorreram predominantemente nas primeiras 48 horas após a oviposição e não houve variação temporal nas taxas de predação. Através de armadilhas fotográficas e observações diretas foram identificadas seis espécies predadoras dos ninhos, não havendo prevalência de nenhuma das espécies. A densidade de ninhos não influenciou as taxas de predação, mas houve correlação negativa entre densidade e tempo decorrido entre a desova e a predação. PALAVRAS-CHAVE. Armadilhas fotográficas; Estação Ecológica do Taim; predador; quelônio; tigre-d'água.

O gênero Trachemys ocorre no continente americano dos Estados Unidos à Argentina (GibBons 1990, Seidel 2002). No Brasil está representado por duas espécies: Trachemys dorbigni (Duméril \& Bibron, 1835) (Emydidae) e Trachemys adiutrix (Vanzolini, 1995) (Emydidae). A primeira, popularmente conhecida como tigre-d'água, representa a distribuição mais meridional deste gênero (ERnst 1990) e ocorre no estado do Rio Grande do Sul; além dos países vizinhos, Uruguai e Argentina. A espécie $T$. adiutrix ocorre no estado do Maranhão, nordeste do Brasil (VAnzolini 1995). O tigre d'água é o quelônio mais abundante do estado do Rio Grande do Sul, ocupando a maior variedade de ambientes (rios, lagoas e banhados) (Lema \& Ferreira 1990).

A biologia reprodutiva de T. dorbigni foi estudada na natureza por KRAUSE et al. (1982) e por BAgER et al. (2007), ambos os estudos foram realizados na Estação Ecológica do Taim. As desovas ocorrem normalmente a partir da primeira quinzena de outubro até a primeira quinzena de janeiro, predominantemente no turno da manhã. São depositados, em média, 12 ovos por ninho, podendo uma fêmea realizar até três desovas em uma estação reprodutiva. Os ovos são elípticos e a casca tem consistência pergaminosa (BAGER et al. 2007). Ovos incubados em laboratório por Molina \& Gomes (1998) eclodiram após um período de 54 a 120 dias. KRAUSE et al. (1982) observaram eclosão após 110 dias, para dois ninhos mantidos no ambiente natural.

Historicamente, muitas populações de T. dorbigni estão sujeitas a uma intensa captura de seus ovos e filhotes para abastecer o comércio de animais de estimação (Lema \& Ferreira 1990, Barco \& Larriera 1991, Bager et al. 2007). No entanto, as conseqüências desta prática sobre as populações são ainda desconhecidas. Alex Bager (obs. pess.) notou que os ninhos de $T$. dorbigni depositados na Estação Ecológica do Taim eram intensamente predados por animais.

A sobrevivência dos estágios iniciais do ciclo de vida de quelônios é geralmente muito baixa (Hamilton et al. 2002). A 
morte dos ovos pode ser atribuída a embriões que não se desenvolvem; morte de embriões parcial ou totalmente desenvolvidos e predação. Este último, no entanto, é apontado como a causa primária de mortalidade (Tinkle et al. 1981). As taxas de predação dos ninhos de quelônios são geralmente altas, mas podem apresentar grande variação. CoNGDON et al. (1983) encontraram uma taxa de predação de $67 \%$ para ninhos de Emydoidea blandingi (Holbrook, 1838) (Emydidae). No trabalho de Butler et al. (2004) as taxas foram de 81,9\% e 86,5\% em anos diferentes, para Malaclemys terrapin (Schoepff, 1793) (Emydidae). Na população de Chelydra serpentina (Linnaeus, 1758) (Chelydridae) estudada por CongDon et al. (1987) a predação dos ninhos variou de 30 a 100\%. Grande variação foi também reportada por Kolbe \& JANZEN (2002) para Chrysemys picta (Schneider, 1853) (Emydidae) (19,7 a 76,2\%).

Os objetivos do presente estudo são: avaliar as taxas de predação dos ninhos de T. dorbigni, variações temporais destas taxas, identificar as espécies que atuam como predadoras dos ninhos e sua importância relativa e avaliar a influência da dispersão dos ninhos sobre as taxas e o tempo até a predação.

\section{MATERIAL E MÉTODOS}

\section{Área de Estudo}

O trabalho foi desenvolvido na Base Santa Marta (BSM), limite sul da Estação Ecológica do Taim (ESEC Taim) (32 $50^{\prime} 09,3^{\prime \prime} \mathrm{W}$ e 52 $\left.38^{\prime} 37,2^{\prime \prime} \mathrm{S}\right)$. A ESEC Taim é uma Unidade de Conservação Federal, localizada no extremo sul do estado do Rio Grande do Sul, Brasil. A BSM está instalada na margem oeste da Lagoa Mangueira (Fig. 1), contígua a fazendas onde têm sido desenvolvidas atividades agrícolas há mais de 20 anos (BAGER et al. 2007). O solo arenoso, de origem Quaternária, suporta uma vegetação herbácea rasteira (CALLIARI 1998). O clima é do tipo Cfa (subtropical úmido) da classificação de Köppen (1948). As estações são bem definidas, sendo os invernos frios e verões com altas temperaturas, com precipitação média anual de $1252 \mathrm{~mm}$. A temperatura do mês mais quente é superior a $22^{\circ} \mathrm{C}$ e oscila entre 18 e $-3^{\circ} \mathrm{C}$ no mês mais frio (NIMER 1989).

\section{Coleta de Dados}

Entre setembro de 2005 e janeiro de 2006 foram realizadas cinco expedições, com duração de cinco a 21 dias, totalizando 57 dias de amostragem e 387 horas de esforço amostral. Foi amostrada uma área de 36 ha em busca de fêmeas de T. dorbigni desovando e ninhos recentemente construídos.

Todos os ninhos encontrados $(\mathrm{n}=93)$ foram identificados com estacas numeradas, sendo alguns $(n=35)$ protegidos com tela visando se evitar a predação. A taxa de predação, tempo de predação e identificação dos predadores foram obtidos a partir dos ninhos não protegidos ( $\mathrm{n}=58)$, já a análise de dispersão e de período reprodutivo também considerou aqueles protegidos. Os ninhos foram monitorados três vezes ao dia (Turno 1: $7 \mathrm{~h}-11 \mathrm{~h}$; Turno 2: 11h01min - 15h e Turno 3: 15h01mim - 19h), em busca de sinais de predação. A noite foi considerada o Turno 4

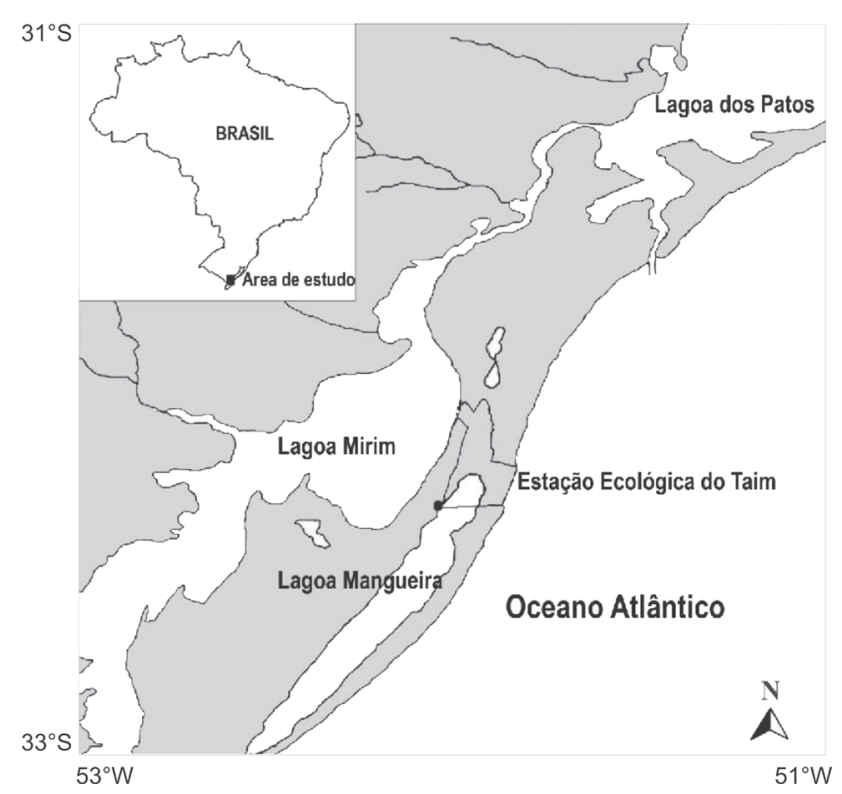

Figura 1. Localização da Base Santa Marta (ponto preto), Estação Ecológica do Taim, no extremo sul do estado do Rio Grande do Sul, Brasil.

(19h01mim - 6h59min). Em relação ao tempo até a predação, as primeiras 24 horas foram divididas do seguinte modo: intervalos de 1 hora, até as primeiras seis horas; seis a 12 horas e 12 a 24 horas. A partir disto foram considerados intervalos diários. Para auxiliar na identificação das espécies predadoras, foram instaladas duas armadilhas fotográficas, em 30 ninhos escolhidos aleatoriamente entre os ninhos que não receberam telas de proteção, totalizando 852 horas de amostragem (armadilha $1=$ 460 h, armadilha $2=392$ h). Observações diretas de predação, realizadas durante os turnos de monitoramento complementaram as informações referentes aos predadores. Para a identificação da freqüência de cada predador, foram considerados tanto registros fotográficos quanto registros visuais. Como foi empreendido esforço diferenciado ao longo do estudo optou-se por padronizar o número de ninhos localizados, dividindo-os pelo número de horas de campo de cada período.

Dados climáticos foram obtidos da Estação Meteorológica instalada na Lagoa Mangueira, fornecidos pelo grupo de estudos do Sistema Hidrológico do Taim, Sítio 7, Instituto de Pesquisas Hidrológicas da Universidade Federal do Rio Grande do Sul (IPH/UFRGS).

\section{Análise Estatística}

Comparações entre proporções e entre taxas foram realizadas através de Qui-quadrado, e quando necessário, foi aplicada a correção de continuidade de Yates. Utilizou-se a estimativa de Kaplan-Meier para se calcular a sobrevivência dos ninhos em função do tempo após a desova. A normalidade dos dados foi verificada com Shapiro-Wilk. O tempo decorrido en- 
tre a ovoposição e a predação ao longo do período reprodutivo foi avaliado através de ANOVA, com prévia transformação dos dados por ln. A comparação entre a idade dos ninhos que cada espécie predou foi realizada através do teste de Kruskal-Wallis, com posterior comparação pelo método Student-Newman-Keuls (Zolman 1993). A curva de suficiência amostral foi calculada no programa EstimateS 7.5 (CoLwell 2005), com 1000 aleatorizações. Para verificar existência de correlação entre a densidade de ninhos e taxa de predação foi utilizado o teste de Spearman, e para a correlação entre densidade e tempo até a predação foi utilizado Pearson. Estes testes foram realizados com o programa BioEstat 4.0 (Ayres et al. 2005).

O perímetro da área de estudo foi demarcado utilizandose gps, as posições de 90 ninhos foram plotadas sobre o mapa da área de estudos gerado pelo programa GPS Trackmaker ${ }^{\circledR} 13.0$ (Ferreira Jr 2005). A área de estudos foi dividida em 36 quadrantes de 1 ha, para a avaliação do padrão de dispersão dos ninhos, através do Índice de Morisita, que foi calculado com o programa Ecological Methodology (Kenney \& Krebs 2000). Todos os resultados foram considerados estatisticamente significativos quando $\mathrm{p}<0,05$.

\section{RESULTADOS}

\section{Período de desova}

Durante os meses de setembro e outubro não foram encontradas fêmeas de T. dorbigni deambulando pelo campo nem vestígios de ninhos predados. O mês de novembro foi o período de maior detectabilidade de ninhos (Tab. I), mas esta diferença não foi significativa $\left(\chi^{2}=0,24 ; \mathrm{gl}=2 ; \mathrm{p}=0,88\right)$ em relação aos outros meses.

Tabela I. Esforço amostral, número de ninhos e taxas de encontro de ninhos de Trachemys dorbigni, na Estação Ecológica do Taim, durante a estação reprodutiva 2005/2006.

\begin{tabular}{lccc}
\multicolumn{1}{c}{ Mês } & Esforço $(\mathrm{h})$ & Ninhos & Ninhos $/ \mathrm{h}$ \\
\hline Setembro & 30 & 0 & 0 \\
Outubro & 60 & 0 & 0 \\
Novembro & 160 & 63 & 0,40 \\
Dezembro & 102 & 28 & 0,27 \\
Janeiro & 35 & 2 & 0,06 \\
\hline Total & 387 & 93 & \\
\hline
\end{tabular}

\section{Predação}

Dos 58 ninhos de T. dorbigni acompanhados, 98\% (n = 57) foram predados. A predação ocorreu em média 1,48 dias após a desova $( \pm 1,44$; mín $=1 \mathrm{~h}$; máx $=7$ dias $)$. As análises empregando Kaplan-Meier evidenciaram que apenas 17,2\% dos ninhos não haviam sido predados decorridos dois dias após a desova; e que ao final da temporada reprodutiva a probabilidade de um ninho permanecer intacto foi de 1,7\% (Fig. 2). A maior freqüência de eventos de predação foi registrada durante as primeiras 24 horas após a desova $(\mathrm{n}=33 ; 58 \%)$, sendo $25 \%(n=14)$ nas primeiras seis horas. As taxas de predação reduziram com o aumento do intervalo de tempo após a desova, e apenas um ninho permaneceu intacto após sete dias, não sendo predado até o final do monitoramento. As taxas de predação mantiveram-se constantes ao longo dos meses em que ocorreram desovas $\left(\chi^{2}=0,74 ; \mathrm{gl}=2 ; \mathrm{p}=0,69\right)$. O tempo médio decorrido entre a ovoposição e a predação também não variou ao longo da estação de desova $\left(\mathrm{F}_{1,55}=0,1081 ; \mathrm{p}=0,74\right)$.

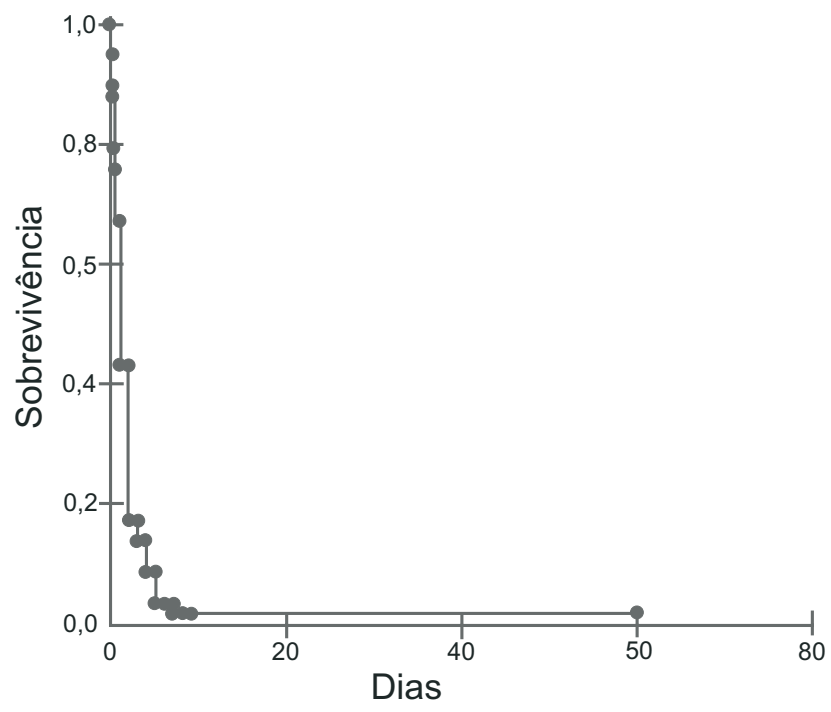

Figura 2. Estimativa de Kaplan-Meier para a sobrevivência de ninhos de Trachemys dorbigni na Base Santa Marta, Estação Ecológica do Taim, na estação reprodutiva de 2005/2006.

Foi verificada diferença na taxa de predação em relação aos turnos de monitoramento $\left(\chi^{2}=13,56\right.$; $\mathrm{gl}=3$; $\left.\mathrm{p}<0,003\right)$, sendo os turnos 4 (noite) e 1 (início da manhã) os que apresentaram maior freqüência de eventos (T4: $\mathrm{n}=40 ; 70 \%$ e T1 n = 10; 17,5\%) (Fig. 3).

\section{Predadores}

As armadilhas fotográficas registraram a atividade de cinco espécies predadoras: Tupinambis merianae (Duméril \& Bibron, 1839) (Teiidae), Conepatus chinga (Molina, 1752) (Mephitidae), Euphractus sexcinctus (Linnaeus,1758) (Dasypodidae), Lycalopex gymnocercus (G. Fischer, 1814) (Canidae) e Cerdocyon thous Linnaeus, 1766) (Canidae). As predações por Caracara plancus (Miller, 1777) (Falconidae) foram registradas apenas por observação direta. Não foi possível identificar a espécie responsável pela predação em $42 \%$ ( $\mathrm{n}=24)$ dos ninhos predados, no entanto, a curva de acumulação de espécies (Fig. 4) mostra que todas as espécies predadoras foram amostradas ( $\mathrm{S}$ obs $=5$ ); sendo que os canídeos foram considerados em conjunto. 

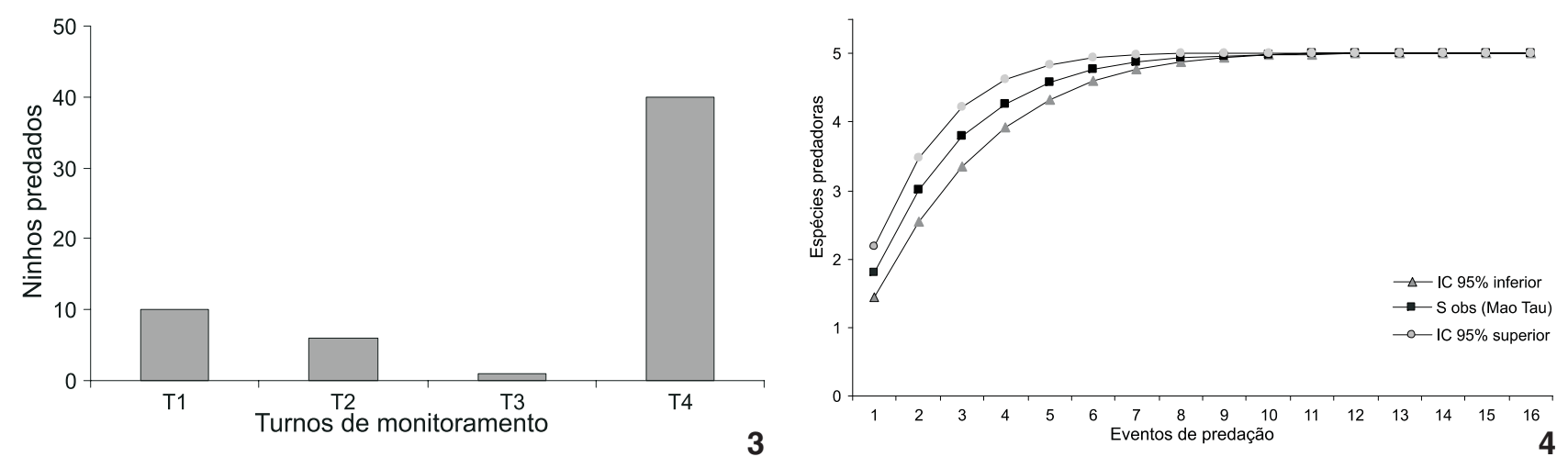

Figuras 3-4. Trachemys dorbigni: (3) número de ninhos de encontrados predados em cada um dos turnos de monitoramento; (4) acumulação de espécies de predadores de ninhos, na Estação Ecológica do Taim, ao longo dos monitoramentos.

A maior freqüência de predação foi verificada para $C$. chinga (31\%), mas esta taxa não foi significativamente maior que a das demais espécies $\left(\chi^{2}=3,21 ; \mathrm{gl}=4 ; \mathrm{p}=0,52\right)$. Houve diferença entre os predadores quanto à idade dos ninhos predados $(\mathrm{H}=19,94 ; \mathrm{gl}=4 ; \mathrm{p}<0,01)$. C. plancus atacou apenas ninhos recentes $(\bar{x}=2 \mathrm{~h} ; \pm 1 \mathrm{~h})$, diferenciando-se das demais espécies $(p<0,05)$, exceto de T. merianae $(p=0,06)$, que predou tanto ninhos recentes (com até 6 horas) quanto mais velhos, com cinco dias após a desova (Tab. II). Em alguns dos ninhos, mais de uma espécie foi fotografada em uma mesma noite, mas não simultaneamente.

\section{Caracterização espacial}

Foram encontrados entre zero e nove ninhos por hectare, com média de 2,52 ninhos $( \pm 2,61)$. O Índice de Morisita mostrou uma distribuição agregada (IM = 1,69; $\mathrm{p}<0,001$ ). Não houve correlação entre a densidade de ninhos e a taxa de predação $\left(r_{s}=0,36 ; p=0,37\right)$. No entanto, em quadrantes onde houve maior densidade, a predação ocorreu em um menor intervalo de tempo $(\mathrm{r}=-0,87 ; \mathrm{p}<0,01 ; \mathrm{n}=79)$.

\section{DISCUSSÃO}

\section{Período de desova}

Em 3/XI/2007, foram encontrados vestígios de ninhos de $T$. dorbigni predados, o que indica que as desovas tenham iniciado na segunda quinzena de outubro. O período de desova verificado neste estudo corrobora BAGER et al. (2007), e discorda de Krause et al. (1982) que citam o período de desova de setembro a fevereiro. Temperaturas baixas durante o mês de setembro (média de $13,8^{\circ} \mathrm{C}$ ) e início de outubro, aliadas a uma alta pluviosidade $(421 \mathrm{~mm})$ podem ter retardado o início das desovas para o final de outubro, quando foram registradas temperaturas médias acima dos $20^{\circ} \mathrm{C}$. Ovos incubados artificialmente em temperatura abaixo de $25^{\circ} \mathrm{C}$ não se desenvolveram (Molina \& Gomes 1998). O início tardio das desovas também deslocou o pico da estação reprodutiva para a segunda quinzena de novembro e primeira quinzena de dezembro, diferente- mente do período citado por BAGER et al. (2007) que foi do final de outubro até início de novembro.

\section{Predação}

A alta taxa de predação sofrida pelos ninhos de T. dorbigni assemelha-se ao encontrado para muitas populações de quelônios de água doce (Congdon et al. 1983, 1987, Bujes 1998, Aresco 2004, Butler et al. 2004); tartarugas marinhas (Brown \& MACDONALD 1995) e mesmo experimentos com ninhos artificiais de quelônios (Wilhoft et al. 1979, Hamilton et al. 2002, MarChAND et al. 2002). Acredita-se que os resultados obtidos reflitam a taxa natural de predação, pois Tuberville \& Burke (1994) e Burke et al. (2005) verificaram que a marcação dos ninhos com artefatos como estacas não aumenta a probabilidade destes serem predados.

A verificação dos ninhos em intervalos de até quatro horas, durante o dia, permitiu um registro detalhado da pressão de predação mesmo nas primeiras horas após a construção destes. A taxa predatória verificada ainda nas primeiras seis horas após a desova demonstrou que os ovos estão sujeitos a predação desde o momento em que são depositados pelas fêmeas. Para ninhos de quelônios, as primeiras 48 horas representam o período mais suscetível a eventos de predação (TINKLE et al. 1981, Congdon et al. 1983, 1987, Burke et al. 2005).

Algumas populações de quelônios experimentam uma predação diferencial ao longo do período de desova. Em alguns estudos, os ninhos depositados nas primeiras semanas estiveram mais sujeitos à predação (Fowler 1979, Kolbe \& JANZEN 2002). No Taim não houve esta variação, a predação mantevese constante, assim como observado por CongDon et al. (1983), considerando-se os meses de novembro e dezembro, já que no mês de janeiro apenas um ninho foi monitorado para análise de predação. O tempo que o predador levou para localizar os ninhos também se manteve constante.

A maior predação durante a noite (T4) pode ser compreendida pelo número de espécies em atividade durante este período (quatro dos seis predadores). Kolbe \& JANZEN (2002) afirmam nunca ter encontrado eventos de predação durante o dia. 
Tabela II. Freqüência de ataques e tempo médio (em horas) decorrido entre a construção dos ninhos de Trachemys dorbigni e a predação, para os ninhos não-protegidos (G1), na ESEC Taim, durante a estação reprodutiva 2005/2006.

\begin{tabular}{llrr}
\hline \multicolumn{1}{c}{ Classe } & \multicolumn{1}{c}{ Espécie } & $\mathrm{n}$ & Tempo $( \pm \mathrm{dp})$ \\
\hline Squamata & Tupinambis merianae & $7(21 \%)$ & $24,0 \mathrm{~h}( \pm 43,0 \mathrm{~h})$ \\
Aves & Caracara plancus & $7(21 \%)$ & $2,0 \mathrm{~h} \quad( \pm 1,0 \mathrm{~h})$ \\
Mammalia & Conepatus chinga & $10(31 \%)$ & $46,0 \mathrm{~h}( \pm 30,5 \mathrm{~h})$ \\
& Euphractus sexcinctus & $4(12 \%)$ & $63,0 \mathrm{~h}( \pm 38,5 \mathrm{~h})$ \\
& Cerdocyon thous e Lycalopex gymnocercus* & $5(15 \%)$ & $38,4 \mathrm{~h}( \pm 20,0 \mathrm{~h})$ \\
\hline
\end{tabular}

* Foram considerados em conjunto.

Uma das explicações para este fato poderia ser o maior intervalo entre as revisões dos ninhos ou a comunidade faunística que atua como predadores ser composta por espécies essencialmente noturnas.

\section{Predadores}

A identificação das espécies predadoras é fundamental para entender a relação predador-presa. No entanto, predadores de ninhos de quelônios geralmente têm sido identificados com base em evidências, como pegadas e marcas de dentes deixadas nas cascas dos ovos (Brown \& MacDonald1995, Maier et al. 2002). Uma alternativa é o uso de armadilhas fotográficas, bastante comuns em estudos de vida selvagem, mas ainda pouco adotadas em pesquisas herpetológicas. Esta metodologia mostrou-se apropriada, registrando cinco das seis espécies que foram identificadas como predadoras dos ninhos de T. dorbigni na área de estudo. Krause et al. (1982) citam estas mesmas espécies como possíveis predadores, baseadas em relatos de moradores do local e funcionários da ESEC Taim.

Os predadores dos ninhos de T. dorbigni, no Taim, têm hábitos generalistas, e muitas vezes, oportunistas, modificando a dieta em função do aumento na disponibilidade de algum recurso (Whitacre et al. 1982, Juarez \& Marinho-Filho 2002, Dalponte \& Tavares-Filho 2004, Donadio et al. 2004, Pedó et al. 2006). A predação por espécies generalistas parece ser um padrão para ninhos de quelônios. Ferreira et al. (2003) relatam que C. plancus e Tupinambis teguixin (Linnaeus, 1758) (Teiidae), além de urubus, predaram ninhos de Podocnemis unifilis (Troschell 1848) (Podocnemidae) no norte do Brasil. Pedó et al. (2006) encontraram cascas de ovos de quelônios em fezes de $C$. thous, no sul do país. Lagartos de médio a grande porte também são predadores comuns de ovos de tartarugas, como Varanus panoptes (Stor, 1980) (Varanidae) na Austrália (BLAmiRes et al. 2003, Blamires 2004). Diversas espécies de canídeos têm sido citadas alimentando-se de ovos de quelônios (Fowler 1979, Congdon et al. 1987, Brown \& Macdonald 1995, Yerli et al. 1997). Mephitis mephitis (Schreber, 1776) (Mephitidae), um similar ecológico de $C$. chinga, na América do Norte também é citado (Bowen \& JANZEN 2005). A espécie mais relatada em estudos dessa natureza, na América do Norte, é Procyon lotor (Linnaeus, 1758) (Procyonidae) (Congdon et al. 1987, Mroziak et al. 2000, Maier et al. 2002, Aresco 2004). No Brasil, Procyon cancrivorus
(Cuvier, 1798) (Procyonidae) também é conhecido por predar ninhos de tartarugas (Bujes 1998, Santos \& Hartz 1999). Na BSM, no entanto, esta espécie não foi registrada, provavelmente por ser mais associada a ambientes florestais (CHEIDA et al. 2006).

Os ninhos foram predados predominantemente até 48 horas após a desova, portanto, não surpreende que não tenha havido acentuada segregação dos predadores quanto à idade dos ninhos consumidos. Este resultado difere do observado por Congdon et al. (1987), onde V. vulpes (Linnaeus, 1758) (Canidae) atacou preferencialmente ninhos com mais de uma semana. Já a predação mais rápida por C. plancus pode ser atribuída ao fato deste ser um predador diurno e visualmente orientado (Sick 1997), enquanto que os demais predadores utilizam principalmente o olfato para localizar a presa (PiAnKa \& VitT 2003, Reis et al. 2006). Em diversas ocasiões durante os monitoramentos, indivíduos de C. plancus foram observados próximos às fêmeas de T. dorbigni, em atividade de desova. Um indivíduo foi observado no chão, a poucos metros da fêmea. Em dado momento, o carcará aproximou-se do ninho e observou seu interior, sem que isto perturbasse a fêmea, que finalizou a ovoposição normalmente. HoRnE et al. (2003) relatam que Corvus ossifragus (Wilson, 1812) (Corvidae) apresenta comportamento semelhante, quando ataca ninhos de Graptemys flavimaculata (Cagle, 1954) (Emydidae).

O lagarto T. merianae, apesar de ter predado principalmente ninhos poucas horas após a oviposição, também foi observado escavando um ninho depositado cinco dias antes.

Feinberg \& Burke (2003) relatam nunca ter encontrado ninhos parcialmente predados. No decorrer deste estudo foram encontrados apenas dois ninhos com alguns ovos ainda intactos. Os registros das armadilhas fotográficas revelaram que alguns ninhos foram encontrados por mais de um predador, da mesma espécie ou de espécies diferentes, na noite em que foram predados. Então, mesmo que um predador não tenha comido todos os ovos de um ninho, os restantes tornaram-se mais vulneráveis a outros ataques, devido aos odores liberados pelos restos dos ovos. MAIER et al. (2002) fotografaram mais de uma espécie visitando o mesmo ninho, e em algumas ocasiões, simultaneamente.

\section{Caracterização espacial}

A influência da densidade de ninhos na taxa de predação varia em diferentes populações. BRown \& MACDONALD (1995) encontraram maior predação associada à densidade de ninhos. 
Na BSM, porém, a predação foi independente do grau de agregação, assim como observado por Fowler (1979). Já o tempo que os ninhos levaram para ser predados foi negativamente correlacionado com a densidade dos quadrantes. Locais com altas densidades de ninhos podem atrair os predadores diminuindo o tempo necessário para localizá-los (VALENZUELA \& JANZEN 2001).

A BSM está localizada em uma área contígua a lavouras de arroz irrigado. Até o ano de 1999 o plantio ocorria até cerca de 20 metros da margem da Lagoa Mangueira. Nesta época, a maior parte dos ninhos localizava-se sobre a área inundada para o plantio (A. BAGER, obs. pess.), sendo que muitas vezes os ovos eram mortos por submersão ou pelo maquinário agrícola. Esta diminuição do número de ovos disponíveis aos predadores pode ter incrementado as taxas históricas de predação. Aliado a isto pode haver um aumento das populações de predadores generalistas que se beneficiam do suprimento extra de vários itens alimentares associados às lavouras e consequentemente, aumentando a pressão sobre populações de alguns tipos de presas (Hamilton et al. 2002). No Taim, a comunidade de predadores que utiliza ovos de tartaruga, em sua alimentação, é formada por espécies amplamente generalistas e que, possivelmente, podem estar se beneficiando da presença de outros tipos de presas, associadas à agricultura, no local. Por exemplo, E. sexcinctus, já foi visto alimentando-se em lavouras de grãos, no estado do Rio Grande do Sul (Silva 1994). O tamanho das populações dos predadores de ninhos de T. dorbigni na BSM permanece desconhecido (exceto para T. merianae - Gisele R. WINCK, Universidade do Estado do Rio de Janeiro, com. pess.), bem como alterações do tamanho populacional ao longo dos anos.

Apesar do estágio de ovo ter uma importância menor na manutenção das populações de quelônios (Course et al. 1987), estas podem se tornar funcionalmente extintas em conseqüência do baixo recrutamento de novos indivíduos. No entanto, estes efeitos podem demorar décadas a serem notados, principalmente quando se leva em conta a presença dos indivíduos adultos (Marchand \& Litvaitis 2004).

O presente estudo demonstrou que a população de $T$. dorbigni na BSM está sujeita a altas taxas de predação, por espécies generalistas, que contribuem de maneira semelhante para a mortalidade dos ninhos. Como registrado para outras populações de quelônios, as primeiras 48 horas após a desova foram as mais críticas para a sobrevivência dos ninhos. A densidade de ninhos não afetou as taxas de predação. Novos estudos para investigar o tamanho das populações de predadores, taxas de eclosão dos ovos e a eficiência de uso de artefatos de proteção contra a predação de ninhos, são necessários. Além desses, sugere-se estudos de longo prazo para investigar a dinâmica populacional dessa espécie, que sofre diversas pressões antrópicas como aumento das áreas de lavoura sobre sítios de desova, intensa pressão de caça para atender o tráfico de animais silvestres, atropelamentos principalmente de fêmeas, em rodovias, além da intensa predação registrada nesse estudo.

\section{AGRADECIMENTOS}

Ao IBAMA pela concessão de licença de pesquisa (053/ 2006). Aos Funcionários da ESEC Taim, pelo apoio logístico. Ao IPH/UFRGS por disponibilizar os dados climatológicos. À Nêmora P. Garcez, Anaide W. Aued, Franciéle Maragno, Leopoldo Telles Neto, Liandro da Rosa e Gisele Winck pelo auxílio na coleta dos dados. FAG é grata a CAPES pela bolsa de estudos.

\section{REFERÊNCIAS BIBLIOGRÁFICAS}

Aresco, M. 2004. Reproductive ecology of Pseudemys floridana and Trachemys scripta (Testudines: Emydidae) in Northwestern Florida. Journal of Herpetology 38 (2): 249-256.

Ayres, M.; M. Ayres Jr; D.L. Ayres \& A.S. dos SAntos. 2005. BioEstat: aplicações estatísticas nas áreas das Ciências Biomédicas. Belém, Imprensa Oficial do Estado do Pará, 324p.

Bager, A.; T.R.O. Freitas \& L. Krause. 2007. Nesting ecology of a population of Trachemys dorbigni (Emydidae) in southern Brazil. Herpetologica 63 (1): 56-65.

Barco, D.M. Del \& A. Larriera. 1991. Sobre la validez de las subespécies de Trachemys dorbigni y su distribucion geografica (Reptilia, Chelonia, Emydinae). Revista de la Asociación de Ciencias Naturales del Litoral 22 (2): 11-17.

Blamires, S.J. 2004. Habitat preferences of coastal goannas (Varanus panoptes): are they exploiter of sea turtle nests at Fog Bay, Australia? Copeia 2: 370-377.

Blamires, S.J.; M.L. Guinea \& R.T.I. Price. 2003. Influence of nest site selection on predation of flatback sea turtle (Natator depressus) egg by varanid lizards in northern Australia. Chelonian Conservation and Biology 4 (4): 1-7.

BowEN, K.D. \& F.J. JANZEN. 2005. Rainfall and depredation of nests of the painted turtle, Chrysemys picta. Journal of Herpetology 39 (4): 649-652.

Brown, L. \& D.W. Macdonald. 1995. Predation on green turtle Chelonia mydas nests by wild canids at Akyatan Beach, Turkey. Biological Conservation 71: 55-60.

Bujes, C.S. 1998. Atividade de nidificação de Phrynops hilarii Duméril \& Bibron (Testudines, Chelidae) na Reserva Biológica do Lami, Rio Grande do Sul, Brasil. Revista Brasileira de Zoologia 15 (4): 921-928.

Burke, R.L.; C.M. Schneider \& M.T. Dolinger. 2005. Cues used by raccoons to find turtle nests: effect of flags, human scent, and diamond-back terrapin signs. Journal of Herpetology 39 (2): 312-315.

Butler, J.A.; C. Broadhurst; M. Green \& Z. Mullin. 2004. Nesting, nest predation and hatchling emergence of the Carolina diamondback terrapin, Malaclemys terrapin centrata, in northeastern Florida. American Midland Naturalist 152: 145-155.

Calliari, L.J. 1998. O ambiente e a biota do estuário da Lagoa dos Patos, p. 13-18. In: U. Seeliger; C. Odebrecht \& J.P. Castello (Eds). Os ecossistemas costeiro e marinho do extremo sul do Brasil. Rio Grande, Ecoscientia, 326p. 
Cheida, C.C.; E. Nakano-Oliveira; R. Fusco-Costa; F. Rocha-MenDes \& J. Quadros. 2006. Ordem Carnívora, p. 231-275. In: N. Reis; A.L. Peracchi; W.A. Pedro \& I.P. Lima (Eds). Mamíferos do Brasil. Londrina, Edição dos Editores, 437p.

Colwell, R.K. EstimateS: Statistical estimation of species richness and shared species from sample. Version 7.5. 2005. Available at: http://www.purl.oclc.org/estimates [Acessed in 01.VI.2006]

Congdon, J.D.; D.W. Tinkle; G.L. Breitenbach \& R.C. Van LoebenSELS, 1983. Nesting ecology and hatching success in turtle Emydoidea blandingi. Herpetologica 39 (4): 417-429.

Congdon, J.D.; G.L. Breitenbach; R.C. van Loeben-Sels \& D.W. TINKLE. 1987. Reproduction and nesting ecology of snapping turtles (Chelidra serpentina) in southeastern Michigan. Herpetologica 43 (1): 39-54.

Course, D.T.; L.B. Crowder \& H. Caswell. 1987. A stage-based population model for loggerhead sea turtle and implications for conservation. Ecology 68: 1412-1423.

Dalponte, J.C. \& J.A. Tavares-Filho. 2004. Diet of the yellow armadillo, Euphractus sexcinctus, in South-Central Brazil, Edentata 6: $37-41$.

Donadio, E.; S. di Martino; M. Aubone \& A.J. Novaro. 2004. Feeding ecology of the Andean hog-nosed skunk (Conepatus chinga) in areas under different land use in north-western Patagonia. Journal of Arid Environments 56: 709-718.

ERnst, C.H. 1990. Systematics, taxonomy, variation, and geographic distribution of the slider turtle, p. 57-67. In: J.W. Gibbons (Ed.). Life story and ecology of the slider turtle. Washington, Smithsonian Institution Press, 368p.

Feinberg, J.A. \& R. Burke. 2003. Nesting Ecology and predation of diamondback terrapins, Malaclemys terrapin at Gateway National Recreation Area, New Jersey. Journal of Herpetology 37 (3): 517-526.

Ferreira-Jr, O.F. 2005. GPS Track-Maker 13.0. Disponível em: http://www.gpstm.com.br [Acessado em 5.VIII.2006]

Ferreira, J.R.; A. Malvásio \& O.S. Guimarães. 2003. Influence of geological factors on reproductive aspects of Podocnemis unifilis (Testudines, Pelomedusidae) on the Javaés Rivers, Araguaia National Park, Brazil. Chelonian Conservation and Biology 4 (3): 626-634.

Fowler, L.E. 1979. Hatching success and nest predation in the green turtle Chelonia mydas, at Tortuguero, Costa Rica. Ecology 60 (5): 946-955.

Gibbons, J.W. 1990. The slider turtle, p. 3-18. In: J.W. GibBons (Ed.). Life Story and Ecology of the slider turtle. Washington, Smithsonian Institution Press, 368p.

Hamilton, A.M.; A.H. Freedman \& R. Franz. 2002. Effect of deer feeders, habitat and sensory cues on predation rates on artificial turtle nests. American Midland Naturalist 147: 123 134.

Horne, B.D.; R.J. Brauman; M.J.G. Moore \& R.A. Seigel. 2003. Reproductive and nesting ecology of the yellow-blotched map-turtle, Graptemys flavimaculata: implications for conservation and management. Copeia 4: 729-738.

Juarez, K.M. \& J. Marinho-Filho. 2002. Diet, habitat use, and home ranges of sympatric canids in Central Brazil. Journal of Mammalogy 83 (4): 925-933.

Kenney, A.J. \& C. Krebs. 2000. Programs for ecological methodology 5.2. Available at http://www.zoology.ubc.ca/ $\sim$ krebs [Accessed in 16.III.2006]

Kolbe, J.J. \& F.J. Janzen. 2002. Spatial and temporal dynamics of turtle nest predation: edge effects. Oikos 95: 538-544.

Köppen, W. 1948. Climatologia. México, Fondo de Cultura Económica, 478p.

Krause, L.; N. Gomes \& K.L. Leyser. 1982. Observações sobre nidificação e desenvolvimento de Chrysemys dorbigni (Duméril \& Bibron, 1835) (Testudines, Emydidae) na Estação Ecológica do Taim, Rio Grande do Sul. Revista Brasileira de Zoologia 1 (1): 79-90.

Lema, T. De \& M.T.S. Ferreira. 1990. Contribuição ao conhecimento dos testudines do Rio Grande do Sul (Brasil) - Lista Sistemática comentada (Reptilia). Acta Biologica Leopoldensia 12 (1): 125-164.

Maier, T.J.; N.M. Marchand; R. Degraaf \& J.A. Litvaitis. 2002. A subterranean camera trigger for identifying predators excavating turtle nests. Herpetological Review 34 (4): 284287.

MarChAND, M.N. \& J.A. Litvaitis. 2004. Effects of habitat features and landscape composition on the population structure of a common aquatic turtle in a region undergoing rapid development. Conservation Biology 18 (3): 758-767.

Marchand, M. N.; J.A. Litvaitis; T.J. Maier \& R.M. DeGraaf. 2002. Use of artificial nests to investigate predation on freshwater turtle nests. Wildlife Society Bulletin 30 (4):1-7.

Molina, F. DE B. \& N. GoMEs. 1998. Incubação artificial dos ovos e processo de eclosão em Trachemys dorbigni (Duméril \& Bibron) (Reptilia, Testudines, Emydidae). Revista Brasileira de Zoologia 15 (1): 135-143.

Mroziak, M.L.; M. SAlmon \& K. Rusenko. 2000. Do wire cages protect sea turtle from foot traffic and mammalian predators? Chelonian Conservation and Biology 3 (4): 693-698.

NimER, E. 1989. Climatologia do Brasil. Rio de Janeiro, IBGE, 421p.

Pedó, E.; A.C. Tomazzoni; S.M. Hartz; A.U. Christoff. 2006. Diet of crab-eating fox, Cerdocyon thous (Linneaus) (Carnivora, Canidae), in a suburban area of southern Brazil. Revista Brasileira de Zoologia 23 (3): 637-641.

PiAnKA, E.R. \& L.J. VitT. 2003. Lizards: windows to the evolution of diversity. University of California Press, Ltd, Berkeley, 348p.

Reis, N.; A.L. Peracchi; W.A. Pedro \& I.P. Lima. 2006. Mamíferos do Brasil. Londrina, Edição dos Autores, 437p.

Santos, M.F. \& S.M. Hartz. 1999. The food habits of Procyon cancrivous (Carnivora, Procyonidae) in the Lami Biological Reserve, Porto Alegre, Southern Brazil. Mammalia 63 (4): 525-530. 
Seidel, M.E. 2002. Taxonomic observations on extant species and subespecies of slider turtles, genus Trachemys. Journal of Herpetology 36 (2): 258-292.

SICK, H. 1997. Ornitologia Brasileira. Rio de Janeiro, Ed. Nova Fronteira, 912p.

Silva, F. 1994. Mamíferos silvestres - Rio Grande do Sul. Porto Alegre, Fundação Zoobotânica do Rio Grande do Sul, $2^{\mathrm{a}}$ ed., 244p.

Tinkle, D.; J.D. Congdon \& P.C. Rosen. 1981. Nesting frequency and success: implications for the demography of painted turtles. Ecology 62 (6) 1426-1432.

Tuberville, T.D. \& V.J. Burke. 1994. Do flag markers attract turtle nest predators? Journal of Herpetology 28 (4): 514-516.

Valenzuela, N. \& F.J. Janzen. 2001. Nest-site philopatry and the evolution of temperature-dependent sex determination. Evolutionary Ecology Research 3: 779-794.
Vanzolini, P.E. 1995. A new species of turtle, genus Trachemys, from state of Maranhão, Brasil (Testudines, Emydidae). Revista Brasileira de Biologia 55 (1): 111-125.

Whitacre, D.; D. Ukrain \& G. FalXa. 1982. Notes on the hunting behavior and diet of the Crested Caracara in northeastern Chiapas and Tabasco, Mexico. Wilson Bulletin 94 (4): 565-566.

Wilhoft, D.C.; M.G. Del Bivaglio \& M.D. Del Bivaglio. 1979. Observations on mammalian predation of snapping turtle nests (Reptilia, Testudines, Chelydridae). Journal of Herpetology 13 (4): 435-438.

Yerli, S.; A.F. Canbolat; L.J. Brown \& D.W. Macdonald. 1997. Mesh grids protect loggerhead turtle Caretta caretta nests from red foxes Vulpes vulpes. Biological Conservation 82: 109-111.

Zolman, J.F. 1993. Biostatisctics: experimental design and statistical inference. New York, Oxford University Press, 343p.

Recebido em 29.V.2007; aceito em 22.XI.2007. 